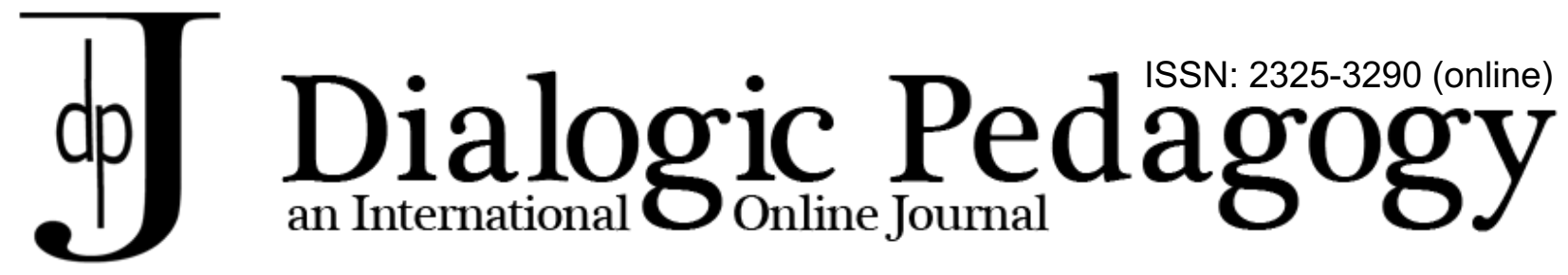

\title{
Initiation, Response, Follow-up and Beyond: Analyzing Dialogue Around Difficulty in a Tutorial Setting
}

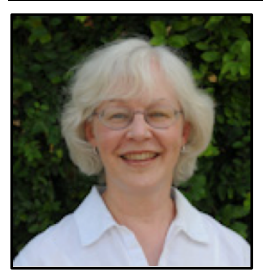

\author{
Elizabeth L. Jaeger
}

University of Arizona, USA

\begin{abstract}
With the advent of Common Core-based assessments, and resulting concerns about academic achievement, more and more students may require the level of instructional intensity tutoring affords. The extent of knowledge regarding the discourse that occurs within the tutoring context is, however, limited. As a result, it is difficult to envision and implement a protocol that incorporates responsive tutor/tutee interaction. This article describes an analysis of discourse patterns that occur as a tutor responded to student difficulty. The study is framed using Bakhtin's concept of dialoguethe ways in which interactions are influenced by the joint speaker/listener identity that is characteristic of interlocutorsand the way this played out in a dialogic instructional context. Excerpts from eight previous tutoring studies served as a foundation for the present research. The primary data source for the analysis was start-to-finish audio-recordings of 40 hours of instruction with two fourth grade readers. After preliminary open coding, overarching categories such as questioning, providing information, and demonstrating strategy use-and more detailed codes within these categories-were applied to the transcripts. Major findings demonstrated that: (a) the tutor's moves were varied and balanced and differed somewhat from child to child, (b) some interactional sequences appeared more effective than others depending on the topic and child, and (c) interactions in this setting differed in important ways from those found in the research literature. I argue here that the dialogic characteristics of tutor/tutee interactions served the children involved and should serve as the basis for additional tutoring protocols.
\end{abstract}

Key Words: dialogue, dialogic pedagogy/teaching, discourse analysis, struggling readers, triadic discourse, tutoring

Dr. Elizabeth L. Jaeger is an associate professor in the department of Teaching, Learning, and Sociocultural Studies at the University of Arizona. She has two primary research interests: (a) understanding and supporting vulnerable readers-that is, those readers who, for a range of reasons including macro-system factors such as race and class, are challenged to learn to read in schools, and (b) exploring the work and experience of "unexpected writers"-from toddlers to academically challenged students to adults dealing with mental illness-who engage in writing for personal fulfillment. Dr. Jaeger has published in a diverse range of journals such as Human Development, British Journal of Sociology of Education, and Literacy Research and Instruction. She can be reached at elizabethjaeger@email.arizona.edu.

$\cos 0380$ 
When instruction is differentiated to meet a child's academic and personal needs, tutoring has the potential to offer the most intense and efficacious learning for readers who struggle (Cohen, Kulik, and Kulik, 1982; Shenderovich, Thurston, \& Miller, 2016). A good deal is known about effective tutoring protocols. Tutors need to collect detailed information about the child's strengths, challenges, and opinions, including informal assessment data (Timmons \& Morgan, 2010) and interviews (Massey, 2007). Also important is basing lessons on student data (Leal, Johanson, Toth, \& Huang, 2004), allowing students to select texts for reading (Mokhtari, Hutchison, \& Edwards, 2010), and employing mediational tools such as lists of steps that support children as they begin to internalize reading strategies (Jaeger, 2017). Historically, however, these studies have failed to forge a link between the results obtained and the quality of tutoring discourse. This is problematic because, as a result, it is difficult to imagine in any detail what quality tutoring looks and sounds like and, therefore, to replicate it.

With more students failing assessments based on the Common Core State Standards (CCSS), it is important to offer tutoring for those students who need it, and to carefully craft tutor-student dialogue, with increased achievement and engagement as the goal. The purpose of this article is to describe and analyze the discourse occurring between a tutor and two students. Specifically, the research study serving as the foundation for this paper examined the difficulties exhibited by vulnerable readers ${ }^{1}$ as they read extended text during tutoring, the responses made by the tutor to those difficulties, and how these responses influenced the tutoring path moving forward. As such, it addresses the following research questions:

- What are the characteristics of dialogue occurring between two fourth grade students and an adult in a study of tutoring interactions? Does this dialogue vary by student?

- In what ways are these dialogue characteristics linked to instructional sequences that end with the child coming to an understanding of the content with which s/he originally struggled? Do these characteristics vary by student?

- In what ways is the instructional context and the dialogue that characterizes the tutoring sessions studied similar to and different from that exhibited in the reviewed theoretical and research literature?

I argue here that the dialogic characteristics of these interactions served the children involved, and differ from that of most tutoring protocols described in the research literature. Bakhtin's (1986) construct of dialogue - a responsive, tension-filled, and collaborative speech genre-is the theoretical frame for this paper; connected to it are the related practices of instructional dialogicality.

\section{Theoretical Framework}

\section{Dialogue}

The term dialogue features prominently in Bakhtin's (1986) seminal essay, The Problem of Speech Genres. He defines dialogue as a responsive exchange between/among interlocutors resulting in shared meaning-making and describes four primary principles of dialogue. First, dialogue is a speech genre. As such, dialogic structures are relatively stable; even specific language is commonly "borrowed" from other utterances. Were this not the case-if speakers and listeners were forced to create new genres for each occasion-communication would be challenging, if not impossible (Bakhtin, 1986). Dialogue serves to craft and re-craft the conditions of a given setting by establishing typical patterns of communication. Second, addressivity and responsivity characterize dialogic chains. Utterances-that is, single speech turns that

${ }^{1}$ I use this term when referring to readers who are unusually sensitive to disruptions in their literacy ecology such as uninteresting texts, inauthentic tasks, and potentially oppressive class, race, and gender factors (Jaeger, 2015). 
serve as the fundamental unit of analysis in human communication-are always directed toward someone (Bakhtin, 1986), and dialogue is, from the outset, a two-way street. A speaker moves from the prior history of utterances on a given topic to her/his own utterance which occurs in anticipation of the listener's response (Wells, 1999). When a remark is made, the person addressed replies in some way (even sitting silently is a response), the initiator follows up, and so on; dialogue has, as such, no beginning or end. As Wegerif (2011) asserted, "(T)he exact meaning of each position in a dialogue depends on succeeding utterances and so can never be closed down" (p. 180). Third, dialogue is fraught with tension and those attempting to communicate need to work with this tension rather than wishing it away. The assumption that the primary goal of dialogue is intersubjectivity without disagreement is erroneous. Conflict is not some unfortunate nuisance, but rather serves to propel dialogue forward (Matusov, 1996). Finally, the purpose of dialogue is collaborative meaning-making. In Bakhtin's (1973) view, "truth" emerges from dialogic practice, rather than residing in the mind of a single individual. As interlocutors interact, they move ever-closer to a shared reality.

\section{Dialogicality in Instructional Spaces}

Educational spaces present as more or less dialogic, so Bakhtin's theoretical work has implications for instruction. Referencing typical classroom instruction, Johnstone (2002) stated that, teachers "decide what will be talked about in what way, and for what purpose" (p. 121). They select content standards and the curriculum employed to facilitate mastery of these standards. They control "the floor" during discussion: proposing topics, asking questions, nominating respondents, and accepting, extending, or rejecting what is said. This type of environment strays far from Bakhtin's (1986) aspects of dialogue. The speech genres characteristics of these spaces constrain, rather than facilitate, generative interaction. In an effort to support children on the path from lesser to greater academic proficiency, tension surfaces, but it is uncommon for this tension to be explicitly noted or effectively employed to promote development. Teachers often anticipate what students will say during an exchange-a form of addressivity-but replies to students' statements tend to be limited in number and unrelated to student comments (Mehan, 1979). Finally, teachers may have thoughtful goals for these discussions, but do not always build a shared meaning with students.

This view of the instructional context may be the most familiar, but we might imagine a classroom or tutoring environment differing, in many respects, from the one Johnstone (2002) described: an environment defined by dialogicality. Such a context is facilitated by a teacher's decision to adopt what Boyd and Markarian (2015) refer to as a dialogic stance. This stance requires of the teacher the ability and willingness to acknowledge the tension associated with classroom power differentials, access students' opinions about the support they need, and engage in self-critique and self-correction. It entails practices of careful listening and purposeful, back-and-forth patterns of leading and following, thereby assisting students to elaborate, analyze, and critique topics of concern. Such talk patterns open spaces for the free-flow of ideas and the inclusion of varied perspectives. This stance facilitates student agency based on "improvisation, creativity, originality, diversity, and uniqueness" (Matusov, 2011). When teachers adopt a dialogic stance, their teaching practice reflects that stance. The primary interactional genre includes question structures to provoke thoughtful answers, answers that provoke student questions, and a question/answer chain supporting a coherent and responsive line of inquiry (Alexander, 2004). Teachers and students embrace tension in this space where problems are posed, disagreements expressed, and paradoxes explored (Matusov, 2009). The overarching goal of instructional practice is collaborative meaning-making (English, 2016).

\section{Literature Review}

In 2014, Mercer produced a review claiming that common instructional talk patterns have the potential to undermine collaborative meaning-making, but that, when teachers adjust their interactive 
patterns to cultivate rather than just assess thinking, student understanding and engagement is positively affected. This claim - that teachers can intentionally alter their instructional discourse- offers important implications for the study of teacher-student talk in general and of this study in particular. In this literature review, I include classroom discourse research both because the literature on tutoring discourse is sparse and because many of the findings about classroom discourse are also relevant for the tutoring setting. Then, I focus on what is known about discourse between tutors and their students.

\section{Discourse and Dialogue in the Classroom Setting}

Tri-fold discourse pattern. An oft-addressed genre of classroom interaction is the ubiquitous trifold pattern referred to by Mehan (1979) as Initiation-Response-Evaluation (IRE) and, by Sinclair and Coulthard (1975), as Initiation-Response-Follow-up (IRF). Researchers have demonstrated that this discourse pattern predominates in classrooms today, as it did at the time of Sinclair and Couthard's original work (Howe \& Abedin, 2013).

Beliefs about the efficacy of this interaction pattern vary. Sinclair and Coulthard (1975) took a descriptive stance toward the phenomenon and chose not to evaluate its effectiveness. In contrast, others adopted a normative position. Advocating for the usefulness of this discoursal genre, Lee (2007) noted that triadic discourse surfaces issues in teaching and learning which require attention; student misunderstandings are, for example, displayed in these interactions and can then be addressed. Nystrand, Wu, Gamoran, Zeiser, and Long (2003) argued that although an evaluative move may hinder dialogue, asking a follow-up question instead facilitates shared understanding and adds coherence to the discussion. Other researchers, however, insisted that, in the triadic model, teachers act as inquisitors (Wood, 1992) and the potential richness of instructional dialogue is inhibited (Mehan, 1979).

Assuming a more balanced stance, Wells (1999) asserted that discussions of talk patterns have been over-simplified and there is room for opened-ended dialogue within an IRF interaction. In a study of classroom interaction characterized by a predominance of triadic dialogue, Wells (1993) found that most discussion topics were, nevertheless, co-constructed. Boyd and Markarian (2015) noted that even if the potential drawbacks of tri-fold discourse are acknowledged, it appears studying the functions of classroom talk rather than just the presence or absence of specific dialogic features, better supports teachers as they strive to listen carefully and respond accordingly, thereby cultivating a collaborative and productive discourse community. They state, "A dialogic instructional stance . . . is a function of how patterns of talk may open up discourse space for exploration and varied opinions" (p. 273), thereby emphasizing the content of the talk (e.g., opinions) as well as its form. In short, it is not the presence of the trifold pattern per se, but its rigid and restrictive implementation that is the issue. If teachers employ IRF in its most openended and dialogic iteration, it can, in fact, facilitate rich discussion.

Types of follow-up moves. Researchers have noted a variety of follow-up moves within the triadic structure; these moves range from less to more interventionist in nature. Edwards (1992) suggested that remaining silent for a few seconds after a child's comment allows for the greatest freedom and agency. The adult can simply repeat or recap what children say (Mercer, 1995) or acknowledge the contribution made (Boyd, 2015), again leaving the door open for children to proceed as desired. Other follow-up options include reformulating or extending a child's response (Mercer, 2004), admitting confusion, or inviting the child to expand upon or inquire about what has been said (Edwards, 1992). More directive moves include asking the child additional questions (Boyd, 2015) and providing hints (Mercer, 2004). Finally, the adult can instruct by providing visual or sentence context support, modeling strategy use or academic language, adding new information, and offering examples. Wells (1999) noted that, whereas third-turn moves are typically unconscious, teachers can consciously attend to these moves and work to alter patterns that 
undermine rather than encourage student participation. This statement is also true in the tutoring setting where both more and less directive moves are appropriate at particular times.

\section{Discourse and Dialogue in the Reading Tutorial Setting}

Recent studies offer recommendations for effective tutoring and the ways in which carefully crafted first-turn moves, attentive listening, and later-turn moves linked to the child's response facilitate meaningmaking in that context. The foundation of these actions includes the importance of detailed knowledge about the tutee (Wittwer, Nuckles, Landmann, \& Renkl, 2010) and the crucial role of differentiated teaching based on that knowledge (Herppich, Wittwer, Nuckles, \& Renkl, 2016). In the tutoring context, teaching and learning can be more closely tailored to meet the individual needs of the child involved and the possibility for true responsivity is more likely. This practice is, however, easier theorized than executed. Referencing work with students in the intermediate grades and beyond, Clay (1987) stated: "(T)he failure of teachers to make marked progress with older failing readers is because they are not able to deliver contingent responses to the pupils' effort. They cannot imagine the response systems the children are working from" (p. 162). This work requires imagination as well as teaching skill.

To explore a possible link between what the literature says about tutoring with what it says about teacher/student dialogue, I reviewed eleven studies, accessed with the search terms tutor and dialogue/dialogic. Each of these studies examined reading tutoring for elementary students with one adult for each one or two children ${ }^{2}$ and included quotes from their interactions. Three studies were subsequently eliminated because they differed in important ways from the protocol for this study; they (a) focused on teaching isolated skills (Juel, 1996), (b) offered no discussion of extended text (Triplett, 2004), or (c) included excerpts dealing with responses to success, but not difficulty (Sableski, 2009). I conducted an analysis of the remaining eight studies (see Table 1), focusing on the degree to which the tutors and students engaged in dialogue as theorized by Bakhtin (1986): patterned yet responsive, sometimes tensionfilled, and conducted in the service of collaborative meaning-making. In some settings, tutors controlled the interactions; in others, the two parties shared responsibility; and in others, students demonstrated considerable agency.

Table 1

Characteristics of Tutoring Studies that Include Excerpts of Discourse About Student Difficulty

\begin{tabular}{lllll}
\hline Study & Population & $\begin{array}{l}\text { Number } \\
\text { of } \\
\text { Children }\end{array}$ & $\begin{array}{l}\text { Tutoring } \\
\text { Interactions } \\
\text { Quoted }\end{array}$ & Other \\
\hline $\begin{array}{l}\text { Pinnell, et al. } \\
\text { (1994) }\end{array}$ & Grade 1 & 403 & 1 & Study of Reading Recovery \\
$\begin{array}{l}\text { Rodgers } \\
(2004 / 2005)\end{array}$ & Grade 1 & 4 & 2 & Study of Reading Recovery \\
$\begin{array}{l}\text { Timmons \& } \\
\text { Morgan (2010) }\end{array}$ & Grade 1 & 4 & 2 & $\begin{array}{l}\text { Tutors were pre-service teachers } \\
\text { Dawes (2007) }\end{array}$ \\
$\begin{array}{llll}\text { Elementary } \\
\text { Newcomer (2010) }\end{array}$ & 3 & 3 & $\begin{array}{l}\text { Volunteers read aloud to children; they } \\
\text { were not positioned as "tutors" }\end{array}$ \\
& elementary & 3 & 3 & $\begin{array}{l}\text { Study of Reading Partners, staffed by } \\
\text { community volunteers }\end{array}$
\end{tabular}

\footnotetext{
${ }^{2}$ In one case, it was referred to as a "small group" with an unspecified number of children.
} 


$\begin{array}{lllll}\begin{array}{l}\text { Hedin \& } \\ (2013)\end{array} & \text { Gaffney } & \text { Unclear } & 7 & \text { Small group tutoring } \\ \begin{array}{l}\text { Almazrouhi } \\ \text { (2007) }\end{array} & \text { Grade 3 } & 1 & 2 & \text { Tutor was also researcher } \\ \text { Lewis (1993) } & \text { Grade 4 } & 1 & 3 & \text { Tutor was also researcher }\end{array}$

Tutor-controlled interaction. Interaction within four of the studies and a subset of a fifth was controlled almost exclusively by the tutor. The first two described research on Reading Recovery, the wellknown tutoring program with an explicit lesson structure. Pinnell, Lyons, Deford, Bryk, and Selzer (1994) included a single quote from tutoring interactions. In response to her struggling student, the tutor provided encouragement and asked the child whether what she had read made sense in context. The other author (Rodgers, 2004/2005) analyzed Reading Recovery lessons by two tutors. She noted that the tutors employed two types of prompts: those focusing on individual words and those directing the student to apply a strategy, such as reading ahead; they varied the help provided in response to student need. Timmons and Morgan (2010) studied pre-service teachers and their tutees. After noting a child's decoding strategy, a less able tutor continued to emphasize that single strategy as she asked one question after another and corrected the child's attempts. Hedin and Gaffney (2013) investigated sixth grade tutees working with doctoral students. Lesson analysis demonstrated that tutors tended to follow set patterns of interaction, rather than responding to what students said and did. Dawes (2007) studied volunteers who read aloud to elementary children during their lunch break, reviewing one hour-long session for each of three partnerships. One of the tutors carefully controlled the academically-oriented interactions and struggled to maintain student engagement.

Balanced interactions. Tutor/tutee discourse within three of the studies was somewhat more balanced. The second partnership in the Dawes (2007) study was one of these. Tutoring excerpts demonstrated that both partners viewed reading as a source of meaning reached through collaboration. Newcomer (2010) studied a tutoring program staffed by community volunteers. Students whose tutors balanced reading and talk and varied feedback as needed posted the greatest gains in reading comprehension. Almazrouhi (2007) tutored a third-grade student, employing a variety of practices to support the boy's literacy growth. In this study, tutor-student dialogue facilitated the use of syntactic and semantic cuing systems and meaning-making.

Student agency in interactions. Finally, two researchers allowed the child more agency in determining the course of their dialogue. The third partnerships in the Dawes (2007) study included a kindergarten child. His tutor allowed him to select the texts they read together and, as a team, they interacted playfully with both the texts and each other. Lewis (1993) recounted interactions with a fourthgrade boy who struggled to make meaning from text. They read and discussed a fable together. Lewis initially rejected the child's interpretation of the moral of the story, but by attending carefully to his explanations, she eventually understood that both she and the child had accomplished what she called "selective reading": attending to the storyline that was easiest for them to follow while discounting other options.

The research literature provides glimpses of what tutoring dialogue looks like. Tutor-child interactions within these studies exhibited some aspects of Bakhtin's (1986) construct of dialogue: most evidenced predictable speech genres, the exchanges ranged from more to less responsive, tension was sometimes present (although it seemed to interfere with, rather than facilitate, communication), and the 
more effective tutors collaborated with the children to construct meaning. There are methodological gaps in this literature, however. None of the studies described above included a full corpus of transcripts nor employed frequency counts and percentages to ensure that the quoted excerpts accurately represented the corpus. In addition, none focus on data excerpts of tutoring discourse occurring in response to student difficulty. Most importantly, the tutoring study described in this paper is the only one I could find that includes a thorough and systematic analysis of the characteristics proposed by Bakhtin (1986) and an explanation of how particular discourse moves led to successful instructional sequences. The study described here reflects this view.

\section{Method}

Guided by (a) findings from the literature, as well as the gaps within them, (b) studies of effective tutoring protocols (Massey, 2007; Mokhtari, Hutchison, \& Edwards, 2010), and (c) the tenets of instructional dialogicality, I developed and implemented a tutoring protocol and conducted an analysis of tutor/tutee interactions. The site for this exploratory, IRB-approved study was an elementary school in a large urban district in a western state which enrolled 350 students. Of these students, $52 \%$ were Latino, $20 \%$ AfricanAmerican, $12 \%$ Asian, and $16 \%$ other ethnicities/no response given; $59.8 \%$ were English learners and $92.7 \%$ of students received free or reduced-price meals. The administrator was fully committed to serving learners who struggle and invited me to support these students. In addition, the students were receiving solid classroom instruction that served as a strong base for my tutoring work.

\section{Participants}

Four fourth-grade students for whom I was the assigned tutor participated in this study. Prior to beginning our work together, I explained the process of tutoring and data collection to the children and their parents and obtained signed consent/assent documents from each of them. Each child was reading about two years below grade level according to the Critical Reading Inventory (CRI) (Applegate, Quinn, \& Applegate, 2008) when the study began in October. This assessment tool which involved a modified miscue analysis procedure was also used to determine the readers' particular literacy needs (e.g., comprehension, decoding, fluency). Tutor-developed follow-up assessments were employed to confirm results from the CRI and to track progress. While I collected and transcribed data for all the students, I selected two, Sam and Bella (pseudonyms), for the analysis that served as the foundation for this paper because they had very different literacy profiles, personalities, and communication styles: Bella was exuberant and chatty, whereas Sam was reserved. Sam was a Chinese-American child, recently reclassified as English proficient. He was a fluent decoder who struggled to construct meaning from what he read. Bella was a Mexican-American English learner. Her decoding and literal comprehension skills were more than adequate, but she struggled with academic vocabulary.

\section{Instructional Design}

The instructional design reflected recommendations from the research literature. It was intended to meet individual student needs and to facilitate dialogue that was responsive and focused on meaningmaking. The design process and session protocol is outlined below (see Jaeger, 2017, for a more detailed description).

I incorporated four major elements in the tutoring design, all of which reflect the construct of instructional dialogicality. First, the instructional design was data-based and differentiated, allowing for dialogue specifically suited to each child (Massey, 2007; Timmons \& Morgan, 2010). An analysis of miscues and retellings from the CRI helped me better understand each child's strengths and challenges (e.g., Sam decoded fluently, but struggled with comprehension, whereas Bella's low-inference comprehension was 
strong, but academic vocabulary and high-inference comprehension proved difficult). An interest inventory facilitated incorporation of students' interests. This assessment data informed the initial differentiated instruction I provided (Leal, Johanson, Toth, \& Chuang, 2004).

Second, a stable, structure with predictable procedures-a speech genre, of sorts-supported our joint efforts to build a safe space for teaching and learning (Lefstein, 2010). A typical tutoring session had two parts. For the first 30 minutes or so, we worked on the goal we had both agreed to, employing a gradual release of responsibility approach (Pearson \& Gallagher, 1983) and a strategy-instruction model similar to that developed by Baumann, Jones, and Seifert-Kessel (1993). For example, Sam and I both agreed he would benefit from work on generating predictions supported by evidence from the text. Then, about 10 minutes' time was allotted for self-selected reading. Because the students did not have a regular SSR time in their classrooms, I felt it was important to provide such a time during our tutorial sessions, allowing them to integrate what they had learned in a given session while reading without interruption. Tutorial sessions occurred two times per week for 40 minutes each, for a total of 30 sessions ( 20 hours) per child.

Third, the protocol was collaborative, goal-directed, and responsive. We made joint decisions about what we would work on when, how, and with what texts (Turner \& Paris, 1995). Then we discussed what "counted" as improvement in that area, set specific goals (Kohn, 1993; Sanacore, 1999), and estimated how long it would take to reach them. As such, these were SMART goals: straightforward, measurable, attainable, relevant (to the child's needs), and time-bound (MindTools, 2017). We regularly revisited these decisions as needed in response to evidence from the ongoing tutorial process (Massey, 2007). While I established this protocol, student cooperation and general enthusiasm confirmed their willingness to engage in it, despite (or because of) the fact that these types of instructional interactions were not part of their prior experience. There were times when Sam withdrew from interaction; I attribute this to his concern about being away from classroom lessons and I took care that he never missed a lesson that particularly grabbed his interest. Bella questioned the structure when she wanted more time for self-select reading; on most occasions, I allowed for this change.

Finally, our sessions were uniformly meaning-focused (Baumann, Jones, \& Seifert, 1993; Fitts \& Gross, 2012; Massey, 2007). Even when the child's goal involved decoding or fluency, our work was contextualized in the reading of extended text. As we read together, I regularly asked whether the text, as read, made sense and, if not, what we might do so we could both understand.

\section{Data Collection}

I collected a range of data including assessments and interviews with the two students, their parents, and teachers (see Jaeger, 2015, for the full interview protocol). Discourse analysis of tutoring interactions is the focus for this paper, however, so the primary data source employed here is start-to-finish audio-recordings of each of our tutoring sessions, transcribed for closer analysis. These tapes provided a rich and relatively objective source of data. They allowed me to hear and analyze aspects of our interaction of which I, as the tutor, might otherwise have been unaware, and bring a critical eye to the analysis process. I consulted interview transcripts and notes taken immediately after tutoring sessions as needed to triangulate findings and shed light on aspects of the session transcripts. For example, during her fall interview, Bella said that she was not a good reader; by spring, she stated that she "sounds like anyone else." This data supported evidence of greater confidence during the tutoring sessions. 


\section{Data Analysis}

Informal analysis of data began immediately after the first tutoring sessions in order to facilitate decisions about lesson planning. I listened to audio within a day or two of each session and developed upcoming lesson plans based on what I heard and saw.

Transcription. Once all tutoring sessions had ended, I formally transcribed the audio-recordings. Cameron (2001) recommends that researchers follow "an approach to transcription that reflects the purposes for which the data has been collected" (p. 39). For example, if the research focus is to describe the tenor of an interaction, conversation analysis, including pauses and voice tone, would serve a crucial function (Sacks, Schegloff, \& Jefferson, 1974). Transcription characteristic of conversation analysis commits the researcher to a fine-grained linguistics analysis. It was my intent to focus primarily on the content rather than the delivery of the tutoring interactions, so as to fully attend to the four aspects of Bakhtin's (1986) construct of dialogue as highlighted in the theoretical frame. To this end, I took Johnstone's (2002) advice that conversation analysis markings might, in the end, distract readers as they explored the content of the exchange. Rather, I produced a word-for-word transcription, adding notes regarding: actions (e.g., reading, writes down a prediction, pauses, laughs), parts of the audio that were indecipherable, and thoughts about what might be going on (e.g., times when I felt a student might be confused).

Initial coding. After transcription was complete I began systematic coding which was, initially, databased (Gibbs, 2007). The unit of analysis was the "conversational turn of talk" (Boyd \& Markarian, 2015). During this open-coding process, I noted several interesting patterns in the ways in which I, as tutor, responded to student difficulty in the follow-up aspect of the IRF sequence.

The next step was to decide where the follow-up move actually began. Attending to Johnstone's (2002) admonishment that choices about discourse boundaries have important implications, I made a strategic decision: although repetitions and discourse elements such "uh-huh" are usually coded in the discourse literature, I chose not to include these aspects in the analysis in the belief that they drew attention away from the heart of the follow-up move. In the following example, "OK, the first place ..." rather than "Which one?" was coded as the third-turn move because "Colony" was the true answer to the question "Any problems?"

(Turn 1) Elizabeth: How about words on that page? Any problems?

Bella: Uh-hmm.

Elizabeth: Which one?

(Turn 2) Bella: Colony.

(Turn 3) Elizabeth: OK, the first place we see colony is right there and this sentence tells you what it means.

During this initial round of coding, I randomly selected and reviewed 15 of the transcripts $(25 \%)$, coding for follow-up moves and for the way in which the interaction ended: (a) with the student coming to an understanding of the strategy or concept with which s/he originally struggled, (b) with me offering a possible answer, or (c) by abandoning an exchange that appeared fruitless. I revisited this subset of transcripts, as well as those remaining, once codes stabilized.

Discourse theory and coding. The second round of coding was concept-driven (Gibbs, 2007). I used terminology as defined by other discourse analysts (Halliday, 1978; Sinclair \& Coulthard, 1975; Wells, 1996). These terms are defined and exemplified in Table 2. 
Table 2

Discourse Terms, Definitions, and Examples

\begin{tabular}{|c|c|c|}
\hline Term & Definition & Example \\
\hline Course & A collection of lesson units & All tutoring lessons conducted for this study \\
\hline Lesson/session & $\begin{array}{l}\text { Instruction in one subject } \\
\text { (e.g., reading) on one day }\end{array}$ & One tutoring session \\
\hline Topic & $\begin{array}{l}\text { A part of a lesson focusing } \\
\text { on one particular reading } \\
\text { process objective }\end{array}$ & The part of a lesson focused on inferential thinking \\
\hline $\begin{array}{l}\text { Sequence or } \\
\text { exchange }\end{array}$ & $\begin{array}{l}\text { All the moves required to } \\
\text { fulfill, or abandon, the } \\
\text { expectations set up by the } \\
\text { initiating move }\end{array}$ & $\begin{array}{l}\text { Reading a segment of text, discussing its content, } \\
\text { and inferring from that segment }\end{array}$ \\
\hline \multirow[t]{3}{*}{ Move/turn } & \multirow{3}{*}{$\begin{array}{l}\text { A contribution to an } \\
\text { exchange made by a } \\
\text { participant in a single } \\
\text { utterance }\end{array}$} & $\begin{array}{l}\text { Initiation move: tutor's question (e.g., Do you have a } \\
\text { prediction there?) }\end{array}$ \\
\hline & & $\begin{array}{l}\text { Response move: child's reply (e.g., I think he will tell } \\
\text { his friend the truth.) }\end{array}$ \\
\hline & & $\begin{array}{l}\text { Follow-up move: tutor's next move (e.g., What } \\
\text { makes you think that?) }\end{array}$ \\
\hline
\end{tabular}

Codes and categories were justified in three of the ways delineated by Constas (1992): (a) rational (have a logical, usually hierarchical, relationship), (b) referential (employed by other researchers), and (c) empirical (exhaustive and mutually exclusive). Listed in order of less to more interventionist, the categories were: moving to a new sub-topic, asking questions, providing information, and teaching. These codes, described in detail in Table 3, were employed to answer all of the research questions. It should be noted here that, while the overarching categories (e.g., information) emphasized the form of turns, the codes themselves (e.g., visual information) are distinguished by content as well. This focus on content forms the link between traditional discourse analysis and dialogic theory.

Table 3

Codebook of Dialogic Moves

\begin{tabular}{|c|c|c|}
\hline Code & Definition & Example(s) \\
\hline \multicolumn{3}{|c|}{ Moving On } \\
\hline MA & $\begin{array}{l}\text { Moving on after } \\
\text { acknowledgement }\end{array}$ & After a child's adequate response: "Ah, OK." \\
\hline MAF & $\begin{array}{l}\text { Moving on, failure to } \\
\text { acknowledge }\end{array}$ & $\begin{array}{l}\text { After child's inadequate response: "OK" -or- tutor just } \\
\text { continues to read aloud }\end{array}$ \\
\hline \multicolumn{3}{|c|}{ Question } \\
\hline QR & $\begin{array}{l}\text { Question: repeat or } \\
\text { paraphrase }\end{array}$ & $\begin{array}{l}\text { After asking "What if the warm water gets up there and } \\
\text { the cold air comes in?" and the child's inadequate } \\
\text { response: "It's warm when it goes up, but when the } \\
\text { cold air comes in, what happens?" }\end{array}$ \\
\hline
\end{tabular}




\begin{tabular}{|c|c|c|}
\hline QN & Question: narrow it & $\begin{array}{l}\text { After asking "Any inference there?" and child's } \\
\text { inadequate response: "Any idea why he might not look } \\
\text { happy?" }\end{array}$ \\
\hline QC & $\begin{array}{l}\text { Question: clarification } \\
\text { requested from child }\end{array}$ & $\begin{array}{l}\text { After the child talks about a character she } \\
\text { (erroneously) believes to be a daughter: "What is the } \\
\text { "her daughter' part?" }\end{array}$ \\
\hline \multicolumn{3}{|c|}{ Information } \\
\hline IG & Information-general & $\begin{array}{l}\text { When child thinks a pilot is a plane: "It's a type of } \\
\text { person." }\end{array}$ \\
\hline IE & Information-example & $\begin{array}{l}\text { When child cannot think of an emotion: "Well, one } \\
\text { example is angry." }\end{array}$ \\
\hline IV & Information-visual & "I bet you can find it right here in the picture." \\
\hline IC & Information-context & "It turns back into ..." \\
\hline \multicolumn{3}{|c|}{ Teach } \\
\hline TS & $\begin{array}{l}\text { Teach-demonstrates/ } \\
\text { discusses strategy use }\end{array}$ & $\begin{array}{l}\text { "So, let's try reading ahead." -or- Tutor re-reads section } \\
\text { for child }\end{array}$ \\
\hline \multirow[t]{2}{*}{ TT } & Teach—telling an answer & $\begin{array}{l}\text { Tutor asks, "Do you know what surface means?" Child } \\
\text { replies, "It's high up." Tutor says, "When surface water } \\
\text { is frozen, water on top is frozen." }\end{array}$ \\
\hline & & $\begin{array}{l}\text {-or- Child asks if she can read aloud and tutor says } \\
\text { she can }\end{array}$ \\
\hline
\end{tabular}

To answer RQ 2, outcomes at the end of a sequence were coded for level of success, with "success" defined as approximating the answer to a closed initiation or responding in a reasonable way to an open-ended initiation. These supplementary codes reflecting sequence outcome were added to the primary codes when they fell at the end of an exchange:

- $(E S)=$ interaction comes to successful conclusion

- $(E T)=$ tutor provides answer after additional moves

- $(E U)=$ difficulty remains unresolved

I was interested in the variation among moves within longer sequences: sequences that, I believe, provided a more valid assessment of the nature of the dialogue than three-move exchanges. I calculated variation in two ways.

- To figure overall variety, I compared the number of move types within a sequence and the possible number of types of moves; so, if there were two QR codes, two TS codes, and one TT code in a sequence, the ratio would be $3 / 5$ or .6 .

- To figure alternation variety, I compared the potential and actual shifts between each tutor move and the ones preceding and following it. If the exchange included seven moves, there would be at least two possibilities for variety, one between Move 3 and Move 5 and one between Move 5 and Move 7. If Move 3 was a narrower question (QN), Move 5 was another narrower question, and Move 7 was providing visual information (IC), the potential move 
changes were three but the actual changes were only two and the variation ratio was .67.

- To answer RQ 3, excerpts from the articles included in the literature review were coded in the same way.

Trustworthiness. Threats to validity and reliability were mitigated by intensive involvement at the research site over the course of a full school year, audio-recording and transcription of 40 hours of tutoring time, a systematic search for discrepant evidence (in this case, attending to both more and less successful interactions), the use of frequency counts and percentages to insure that quoted excerpts were representative of the data corpus as a whole, and comparison between collected data and that from similar studies in the research literature. Reliability was established via both intra- and inter-rater coding. As recommended by Hedin and Gaffney (2013), 25\% of the tutoring sequences were randomly selected and recoded after a week's delay; intra-rater reliability was .99. An additional $25 \%$ were also selected at random and re-coded by a trained graduate student, resulting in inter-rater reliability of .91 . These supports for validity and reliability added to the trustworthiness of the findings obtained.

\section{Findings and Discussion}

Findings for the three research questions are presented in this section. Each claim is supported by excerpts from the transcribed data and, in the case of $R Q 3$, from the tutoring dialogue reported in the reviewed research literature. I employed four criteria when selecting quotes that would best serve my analytic focus (Jacob, 1987): (a) first and foremost, the excerpts were reflective of the data as a whole; (b) on average, quotes included approximately five moves which was the mean for all sequences in the data; (c) on the other hand, the excerpts were long enough to capture the essence of each interaction; and (d) the number of quotes selected from each child is similar.

Findings from the three research questions reflect Bakhtin's (1986) construct of dialogue. The tutoring I conducted for this study exhibited all aspects of this construct (RQ1). The instructional interactions served as a form of speech genre in that the types of moves were finite-only ten in number-and nearly all sequences were three to nine moves in length. The exchanges were, by definition, responsive; until the beginning of a new sequence, each partner replied directly to the other's question or comment. Because the analysis process focused on response to student difficulty, our interactions included an inherent tension, and disagreements about these difficulties surfaced in the interactions. Most importantly, efforts at meaningmaking drove all our work together. Many of these exchanges ended successfully ( $R Q 2)$. Sequences from studies in the literature tended to be less dialogic than those in the study described here (RQ 3$)$.

\section{What are the Characteristics of Dialogue Occurring in the Tutoring Sessions?}

Across the tutoring interactions from this study, commonalities existed among third-turn and later moves, sequence lengths, and turn variety. Nevertheless, there were differences between interactions with the two students.

Third-turn and later moves. Third-turn and later moves varied depending on the type of move found in the first-turn position; this finding highlights their responsive quality. When first-turn moves were relatively broad in scope (e.g., during a story in which mysterious noises frightened the main character, "Do you have a [personal] connection to that part?"), follow-up questions were often narrower (QN) (e.g., "Have you ever heard strange noises and not known what they were?). This quality served the meaning-driven objective of the exchange. Providing an answer for the child (TT) was more common than I might have hoped, but this practice served to advance the dialogue at points where earlier moves had been less effective and becoming bogged down in detail was a clear risk. On the other hand, given that increased strategy awareness and use was a major goal for the tutoring project, additional strategy demonstrations- 
or more frequently asking the child what strategy was appropriate for a given situation-might have increased the likelihood that the child would have achieved the goal with less direct help. Repeated/paraphrased questions (QR) were much more common in Move 5 or higher (51 versus 12 instances in Move 3). They were typically employed after information was provided, allowing the child to return to the same or similar question with new knowledge.

Except for the MAF move (no verbal acknowledgement-moving on in the sequence-4\%), third move responses were relatively balanced. Offering the child further information ("This is an example of not a confusing word but a confusing idea.") accounted for $28 \%$ of responses, followed by asking a follow-up question ("So, how does egg sack relate to the word mate?"-25\%), providing an answer ("It's a kind of knife."-22\%), and demonstrating/ commenting on strategy use ("Let's read to the end of the sentence."$21 \%)$. Among information types, general verbal information predominated and narrowing questions were more common than other types of questions (see Table 5). The balanced nature of these follow-up moves reflects Wegerif's (2011) assertion that "in lived reality we experience a continuum between more monologic and more dialogic voices" (p. 188). An example of the former would be providing an answer and of the latter asking the student to clarify her/his remarks before proceeding.

Table 5

Frequency of Third Moves

\begin{tabular}{|l|l|l|l|l|l|l|l|l|l|}
\hline MA(F) & QR & QN & QC & IG & IE & IV & IC & TS & TT \\
\hline $4 \%$ & $6 \%$ & $17 \%$ & $2 \%$ & $17 \%$ & $3 \%$ & $5 \%$ & $3 \%$ & $21 \%$ & $22 \%$ \\
\hline
\end{tabular}

Sequence length. In a nod to the genre-driven aspect of dialogue, the average number of moves per sequence was 4.8 , with a range of 3 to 15 . That is, a typical sequence consisted of a tutor or child initiation, followed by a response, a follow-up, another response, and a final follow-up. By the end of the sequence, the student offered an appropriate response, the tutor provided a possible answer, or the conversation was abandoned. The 4.8 figure contrasts quite dramatically with that found by Cromley (2005), the only other researcher who measured numbers of moves. She found an average of 19.6 moves in response to student difficulty, but this difference may be due to the fact that the tutors in Cromley's research were working with adults who were, potentially, more reflective and communicative than the fourth graders in this study.

Sequence variety. When measured in two different ways, interactions had high rates of variety, further evidence of responsivity. First, moves varied across sequences. It was unusual to see one type of move repeated over and over. When comparing the actual to the possible level of variety across moves (explained in more detail in the Method section), the mean rate of overall variation was .67; that is, if a sequence involved three tutor moves, it was uncommon for more than two to be of the same type. Second, frequent changes occurred from move to move. The mean number of changes across sequences was .79; in other words, when shifting from one tutor move to the next, $79 \%$ of the time, on average, the next move was of a different type than the previous move. It was highly unlikely that the child would be, for example, asked one narrow question after another. This excerpt is an example of a high-variety sequence in which Bella recognized the lack of helpful context for a word she wanted to learn:

\section{Elizabeth: (implied first move) $)^{3}$ Are you having any trouble?}

${ }^{3}$ For purposes of this analysis, the tutor always has the odd and the child the even turns. In this system, when the child initiates there is always an implied first move-generally the primary focus for the sequence. 
Bella: I don't know what aphids (are).

Elizabeth: Let's keep going to the end of the sentence. (Move 3-TS)

Bella: [reads on]

Elizabeth: So if we say aphids (are kept) the way human farmers keep cows, does that tell

Bella: I don't know. you what an aphid is? (Move 5-IG and then QR)

Elizabeth: Right, it doesn't. So, let's go back and try RE-reading. (Move 7-TS)

Bella: [re-reads]

Elizabeth: So, an aphid is . . (Move 9-IC)

Bella: [pause] An insect.

In this sequence, tutor moves shifted from strategy demonstration to general information, paraphrased question, strategy demonstration, and providing sentence context. Thus, the sequence exhibited a more conversational tone than would have been the case if tutor moves were less varied (see negative examples in the findings section for the third research question).

Variations by student. My third-turn and ensuing moves differed in response to each student's needs. It was more common for me to provide Bella with an answer after she expressed confusion than was the case for Sam ( $28 \%$ of the time as compared to $16 \%)$. It is likely that I responded in this way because she struggled more with academic vocabulary, both on the CRI and in our ongoing work together; when vocabulary was the focus of our work, our conversations went on at greater length, but at other times, helping her with a word meaning allowed her to focus on the topic at hand. For example:

Elizabeth: (implied first move) Any words you are struggling with?

Bella: [reads] Clouds sweep across the sky. Clouds sweep?

Elizabeth: Because there's nobody sweeping, is there? It just means moving. (Move 3IG and then TT)

Note: The primary instructional focus at this point was reading to [dis]confirm prior knowledge, rather than vocabulary; as such, providing an answer to a word meaning query seemed appropriate.

With an alternative meaning for sweep in hand, Bella could grasp the remainder of the description.

Connecting personal experiences to the text he was reading was an area of focus for Sam but not for Bella. This finding may explain why narrow questions, used to focus his thinking, and non-responses, signaling that further discussion would not be beneficial, were more common in my follow-ups with Sam than with Bella. For example, this sequence in which a more specific question assisted him to access prior experience:

Elizabeth: Is there anything in the story that reminds you of something in your own life?

Sam: ... No.

Elizabeth: Has there ever been something that you should have known that no one told you about ... like they kept a secret from you? (Move 3-QN)

Sam: My friend he kept the secret that he was going to move?

The success Sam achieved in this sequence allowed him to better understand the motivations of the character in the story he was reading.

Dialogic Pedagogy: An International Online Journal | https://dpj.pitt.edu

DOI: 10.5195/dpj.2019.195 | Vol. 7 (2019) 
The two children also differed somewhat in the average length of interactional sequence. Bella, a more talkative child, averaged 5.2 moves per sequence. Sam, on the other hand, was more reserved and averaged 4.2. As he warmed to our work together, however, Sam-like the child in Almazrouhi's (2007) study - became more communicative. His average sequence length during the first half of our lessons was 3.7 turns; this number rose to 4.9 in the second half. The following two sequences demonstrate the difference in our interactions over time:

\section{From Lesson 4:}

Elizabeth: What makes you think that (the ghost would appear)?

Sam: Uh, because I think the ghost was hiding somewhere else.

Elizabeth: Ah, OK [writes down his response before moving on] (Move 3-MA)

\section{From Lesson 24:}

Elizabeth: How do you think they know that the moon was once part of Earth?

Sam: ... I don't know.

Elizabeth: Well, let's say I hit the bottom of my shoe with a knife and a chunk flew off [demonstrates]. That's kind of what happened with the moon, right? How would we know that that chunk was once part of my shoe? (Move 3-IV and then IG and then $Q N$ )

Sam: Uh, because it got off your shoe?

Elizabeth: OK, but let's say all we had was the shoe and my chunk? How would we know that that chunk was once part of my shoe? (Move 5-QN and then another QN)

Sam: Because it's made of your shoe?

In seeking an answer to the first research question, I found that follow-ups, at the third-turn juncture and beyond, were both varied and relatively balanced. On average, sequences lasted about five turns, and it was unusual for moves to be repeated over and over. These findings should inform the crafting of instructional sequences. Nevertheless, follow-ups differed somewhat by child and by area of focus, as did mean sequence length, so it is also important to vary those sequences as needed to meet the needs of individual students. For example, more "telling" might be appropriate for a child who is working to strengthen academic vocabulary and probing questions for less expressive students.

Interactions in our tutoring sessions reflected instructional dialogicality. On one hand, they demonstrated the overall predictability characteristic of a speech genre and this quality provided a dependable framework for learning. Although our conversations demonstrated variety, certain kinds of exchanges-question-after-question-after-question sequences, for example-did not occur. Likewise, the students' overall attentiveness evidenced, I believe, their sense that I was there to listen to them and respond accordingly, even (maybe especially) when tension around the difficulty they were experiencing occurred. Teachers and researchers often view vulnerable readers as a monolithic group (Buly \& Valencia, 2002) and this attitude undermines their progress. Sam and Bella were very different children and responsiveness to those differences supported their growth in the tutorial setting. Finally, the meaningdriven nature of our work together offered an approach to reading that focused on understanding texts at deep levels rather than overemphasizing mastery of isolated skills as is common in many tutoring contexts (Samson, Hines, \& Li, 2015); this is a key piece in grasping why our collaboration was productive. 


\section{In What Ways are Dialogue Characteristics Linked to More and Less Successful Instructional Sequences?}

Some tutoring sequences were more successful than others. A sequence was considered successful if, for example, the child figured out the meaning of an unknown word, generated a reasonable inference, employed a helpful reading strategy, offered a prediction or an opinion, or logically justified those responses. A less successful sequence ended when I provided an answer for the child or went on to another sequence, leaving the discussion unresolved.

The length of interactional sequences and the type of third-turn move seemed to affect the likelihood of end-of-exchange success. Too few (three moves) or too many (13 or more moves) were less likely to end successfully. Overall, follow-up questions were more likely, and providing further information was less likely, to lead to success. Differences existed, however, between the two children. For Bella, providing examples and visuals (e.g., referencing pictures in the text) and asking her to clarify comments I failed to understand was particularly helpful. Sam was most responsive to follow-up questions, especially those reflecting a movement from a more abstract initial move (e.g., Does this part remind you of something?) to more concrete one (e.g., Have you ever ... ?). But a key calculation called the import of end-of-sequence success into question. Only $51 \%$ of tutoring sequences ended with students coming to a reasonable conclusion on their own. This finding was surprising given that both children made over two year's growth on the CRI (Jaeger, 2017).

Predictably, students experienced the least success during sequences addressing their areas of greatest challenge. For example, Sam's success rate in sequences dealing with comprehension monitoring, his greatest challenge, were at $28 \%$ as compared with $55 \%$ overall. Including work with topics that came more easily (e.g., distinguishing important information for Bella and generating opinions for Sam) was important, but it would have been inappropriate to abandon work in areas of greater challenge to produce a higher sequence success rate, because it was in these moments of struggle that we built meaning together. Some of our most valuable interactions, like that below, ended with a telling (TT) move; in fact, awareness that there are times when seeking information elsewhere is a necessary choice was the intent of this conversation:

Elizabeth: What is a rodent?

Bella: Is these baby rodents?

Elizabeth: Those are rodents. Let me give you, let me draw you one of my little diagrams here [draws a concentric circle diagram]. So, here's capybaras (in the inner circle) and here's rodents (in the outer circle). All capybaras are rodents. (Move 3-IG and then IV and then IG)

Bella: All.

Elizabeth: Uh-hmm. Just like all dogs are mammals. Every dog is also a mammal, but is every mammal a dog? (Move 5-IG and then QN)

Bella: No.

Elizabeth: But there's NOTHING (in the text) that tells us what a rodent is and it doesn't work to skip it. So, this is a time that if you needed the meaning of that (rodents) you'd just have to get help. A rodent is an animal that chews on things like mice, rats, rabbits-those are the ones I can think of. (Move 7IG and then TS and then TT)

In this example, absent helpful context clues, we worked hard together to co-construct a potential meaning. I provided the specific word meaning not evident in the text. 
Some conclusions can be drawn about which types of interactions were most beneficial. Sequences of moderate length were more likely to end successfully than short or long ones. I infer that a brief sequence of interactions failed to allow for the extensive thought necessary to come to a reasoned conclusion, whereas it was difficult for students to follow the chain of thought in a very long sequence; both types of sequences, then, interfered with collaborative meaning-making. In general, follow-up questions were the most effective third-turn move, likely because these questions prompted students to think more carefully about their initial responses. The two students differed as to which third-turn moves seemed more effective. Bella made best use of visuals and examples. This preference is likely to be related to her vocabulary challenges, as defining new words might benefit most from these types of moves. Sam benefitted most from questions, especially those that were narrower and more concrete than the initial move had been; these turns were most likely to push him toward an understanding of the text, his greatest challenge. In the end, however, the concept of "sequence success" had less explanatory power than expected. I came to understand that the result of any individual sequence was not crucial. Of course, repeated lack of success at the sequence level would have undermined morale, but the children rarely demonstrated frustration and remained engaged and motivated in the face of "failure." That is, the tension in our dialogue did not seem to bother them. Even if unsuccessful by traditional standards, our responsive instructional conversations facilitated deeper understanding, the primary purpose of the tutoring.

\section{In What Ways is the Instructional Context and the Dialogue that Characterizes the Tutoring Sessions Studied Similar to and Different from that Exhibited in the Reviewed Theoretical and Research Literature?}

Similarities. The protocol for this study reflected both theoretical and research literature. It was designed to explicitly acknowledge the tension resulting from the power differential between tutor and student (Bakhtin, 1986) -and including efforts to diminish that differential by co-developing lesson content-with the intent to foster student agency. For example, we co-selected texts for the instructional sequence and the children chose the texts they wished to read during self-select reading time (Dawes, 2007). Instruction was differentiated based on initial and follow-up assessments (Herppich, Witwer, Nuckles, \& Renkl, 2016). Listening to lesson audio immediately after each session, allowed for self-critique and self-correction in my practice (Boyd \& Markarian, 2015).

It appeared that the enacted curriculum largely reflected the plan's intent. There is evidence in the data for many of the instructional aspects which Boyd and Markarian (2015) argue are part of what they term the dialogic stance. As evidenced from tutorial excerpts:

- Within the IRF sequence that characterized the dialogue, most was co-constructed (with Sam on p. 14; Wells, 1999)

- Chains of questions and answers led to responsive interactions (with Bella on p. 13; Alexander, 2004)

- Follow-up moves ranged from silence (p. 21; Edwards, 1992) to asking additional questions (with Sam on p. 14; Boyd, 2015) to providing hints (with Bella on p. 15; Mercer, 2004)

Differences. Important differences exist between the excerpts included in the studies cited in the tutoring literature and those found in data from the current study. In the study described here, third-turn moves were more closely balanced (see Table 6). "Telling" moves appeared less facilitative than others and one might imagine that a lower percentage of such moves would be ideal; but, when used in moderation (as was the case in this study), they were useful. This finding was particularly true in the case of word meanings (Chazan \& Ball, 1999) which were, along with literal comprehension, the topics in which I 
employed telling most frequently. In the case of the reviewed literature, moves were less balanced, with asking questions and providing information predominating.

Table 6

Percentages of Third-Turn Moves in Current Study and Research Literature

\begin{tabular}{llllll}
\hline & Questions & Information & $\begin{array}{l}\text { Strategy } \\
\text { Instruction }\end{array}$ & $\begin{array}{l}\text { Telling an } \\
\text { Answer }\end{array}$ & $\begin{array}{l}\text { Moving On } \\
\text { W/out Closure }\end{array}$ \\
\hline Current Study & $25 \%$ & $28 \%$ & $21 \%$ & $22 \%$ & $4 \%$ \\
Literature & $39 \%$ & $32 \%$ & $18 \%$ & $11 \%$ & $0 \%$ \\
\hline
\end{tabular}

Further, although the amount of change from move to move was similar (.79 in this study as compared with .75 in the literature) the overall variety differed (.67 in this study and .54 in other research). In other words, in the study described here, only about one-third of the moves in a given sequence were repeated again in that sequence; about half of the moves were repeated in studies described in the research literature (see the Method section for more detailed explanations). These repeated follow-up moves resulted in discourse that read more like an interrogation, as is the case in this excerpt from a tutor in Newcomer's (2010) study:

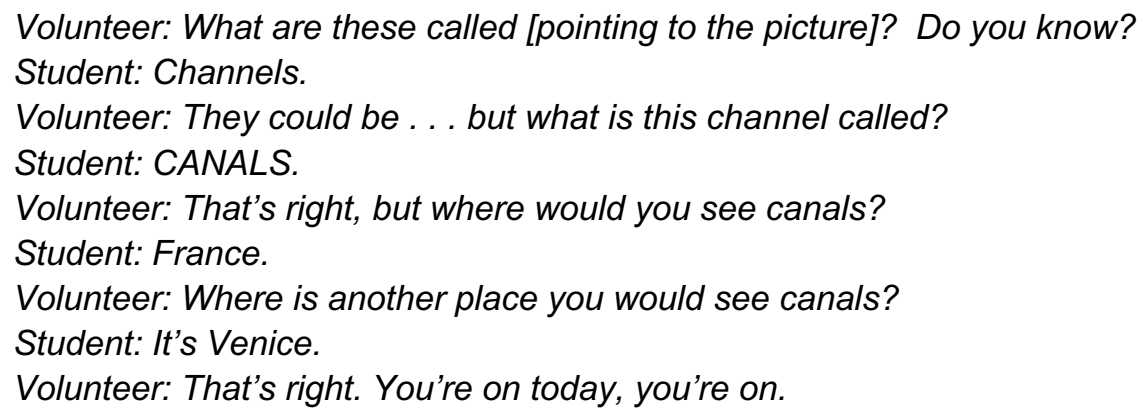

In this exchange, the student exhibited an understanding of isolated facts, but did not experience actual dialogue.

The primary goal of the study reported here was to cultivate strategy use leading to more thoughtful reading. The ratio of closed to total interactions (.68) appeared to emphasize the former over the latter and the overall tutoring process would, no doubt, have been improved by including more open-ended and responsive discussions. This imbalance was, however, more extreme in the reviewed literature: $85 \%$ of sequences were closed interactions, with only one excerpt focusing on predicting and three on formulating opinions. Typical were interactions such as the following from Hedin and Gaffney (2013), consisting of one closed question after another (line structure changed to fit my analytic model):

Ms. Hester: Can you explain what the phases of the moon are?

Marcus: [30 second pause]

Ms. Hester: What would be a different word than phases that you could use there?

Marcus: Rotate?

Ms. Hester: Could it be rotate of the moon?

Marcus: [15 second pause]

Ms. Hester: Which would work better, the rotate of the moon, or the shapes of the moon? 


\section{Marcus: Shapes.}

In comparing the study described here to those in the tutoring literature, two major differences emerged. First, moves in this study exhibited greater variety, whereas excerpts from the literature often had an inquisitional quality to them. The interaction quoted above between Student and Volunteer is a prime example of a constraining speech genre which undermined any responsiveness that might have occurred. Despite the tutor's concluding praise, the student's single-word replies seemed to exhibit an unproductive tension. Second, open exchanges were more evident in this study than in the reviewed literature. As was the case in the exchange between Marcus and Ms. Hestor, there was no room here for inference, interpretation, or creative exploration, but plenty of unresolved tension. In combination, the tutoring interactions with Bella and Sam exhibited a more meaning-driven, responsive, and conversational tone.

\section{Conclusion}

The research literature describes successful tutoring protocols. Such protocols typically involve the collection and application of student data, collaborative text selection, and use of mediational tools. Nevertheless, researchers have failed to analyze, in any substantive and systematic way, instructional conversations occurring during tutor-tutee interactions-the gap addressed in this paper. Absent from the literature are studies including an analysis-employing frequency counts to insure accurate representation - of a full corpus of tutoring transcripts, as well as studies accounting for student differences and the affect these differences have on tutor-tutee interactions. As a result, readers of such research may struggle to gain a clear sense of what these conversations sound like and to craft future interactions more effectively.

Framed by the Bakhtinian (1986) construct of dialogue, this paper describes an analysis of instructional interactions during tutoring, specifically those occurring in response to student difficulty. Consistent with traditional discourse analysis procedures, I analyzed forms of interaction (e.g., providing information). To more clearly connect to the construct of dialogue-and to resolve the tension between the sometimes problematic tri-fold discourse pattern and the theory of dialogic stance-the content of such forms (e.g., providing visual information) was also addressed.

I argue that these exchanges exemplified key aspects of dialogue: they were genre-based, responsive, tension-laden, and meaning-driven. As such, the interactions supported two vulnerable readers as they constructed meaning from text. Due to the CCSS's higher achievement expectations, more and more students will require tutoring if they are to meet those expectations. The merit of the study lies in the way it presents a detailed picture of instructional dialogue, a picture that aids in the development of more valuable tutoring practices for a range of students. In fulfilling this purpose and defending this argument, the study contributes several findings to the literature.

In the model presented here of a tutoring protocol resulting in strong gains for the children who participated, moves that came as follow-ups to the child's initial response-acknowledgement of student response (or lack of same), questions, information, strategy demonstrations/discussion, and provision of a possible answer-varied based on the first-turn move; for example, initially broad questions tended to precipitate narrower questions after the child struggled to respond. Other than continuing with no response, the frequency of moves was nearly equal. The average number of moves within a single sequence was approximately five; in most cases, it did not take long to resolve our discussion or, if conversation stalled, to move on. When looking across the whole range of moves making up a sequence, I discovered considerable variety; it was uncommon for a child to be peppered with question after question or provided with more information than s/he could constructively employ; thus, the interactions took on the flavor of 
dialogue. As tutor, I tended to reply to each child's response in different ways: typically, questions for Sam and information for Bella. Because just over half of all sequences ended with the child coming to an appropriate resolution - or, in many cases, one of several appropriate resolutions-it seems that finding the perfect way to react to a child's comment is less crucial than expected. Attending carefully and replying in ways reflecting interest and attention appear more important. This conclusion confirms Newcomer's (2010) finding that students whose tutors varied their replies to students' statements achieved stronger comprehension gains. In comparison to the tutoring excerpts found in the research literature, those in this study were more varied, more balanced, and more open in orientation.

Gee (2011) recommends an approach to discourse analysis that looks at the narrative of a given setting; he suggests, "For any communication, we want to ask what typical stories . . . the words and phrases of the communication are assuming and inviting listeners to assume" (p. 171). The instructional context most evident as one reads the research literature is one in which tutors unilaterally decide what is studied and how. Such environments diminish student agency and may lead to a passive reader identity. They exhibit a limited range of follow-up moves, falling into a sort of tutoring script rather than responding intentionally to what children say in the moment. Almost without exception, interactions progress, without wavering, toward a pre-determined outcome with little room for inference, opinion, or personal connection. It is as if the fact that children are struggling with the so-called "basics" supersedes any notion that they might have something interesting to say.

The instructional context embraced by this study is, by and large, quite different. The overarching goal of strategy use as a bridge to powerful thinking is clearly established by the tutor, but sub-goals-the ability to infer, gain word meaning from context, or offer justifiable reasons-are negotiated with students. Follow-up moves, at the third-turn position and beyond, come in response to what children say and what they do not say, depending both on what the tutor hears in the moment and what s/he has come to learn about the child over time. As educators, we learn these moves from studying the instructional conversations around us. This is the tutoring "story" vulnerable readers deserve: one that supports their agency and positions them as engaged readers and conforms to a rich definition of dialogue.

The study provides instances of dialogue conforming to Bakhtin's (1986) principles. The tutoring interactions reflected a speech genre of sorts. There occurred few lengthy monologues and, whereas exchanges differed by child, with Bella assuming a more equitable role in the dialogue than did Sam, the bits of transcripts included here exhibited a relatively conversational tone. As I spoke to the students, I often anticipated what they might say in reply - exemplifying the characteristic of addressivity - but I attempted to respond to what the child actually said, rather what I imagined s/he would say. Our interactions were, at times, tension-filled. There were moments when students felt frustrated by my lack of clarity and I by their failure to assert what they knew or felt, but this tension was often generative. Students could surface confusion and expect me to allay that confusion-either by answering questions directly if they were stymied or leading them down a path intended to support them in finding answers of their own. These interactions serve as evidence of collaborative meaning-making.

\section{Limitations}

These findings are limited in several ways. First, and foremost, tutoring sessions for only two participants were reviewed for this study and, as such, findings remain exploratory rather than readily generalizable. Second, there are limitations associated with my dual role as both tutor and researcher and, as a result, the need to depend heavily on audio-recordings for data analysis. Although these recordings were useful, they captured only language-based exchanges; I attempted to take notes about facial expressions and body language but my ability to do so was limited. As such, I certainly missed some of the 
nuances of these interactions, nuances that might affect study findings. On the other hand, I brought to tutoring a wealth of experience helping vulnerable readers: experiences that allowed me to design a protocol which built on students' strengths and supported their agency as learners. In the end, I decided that conducting the tutoring myself was the best choice.

The remaining limitations reflect coding decisions. I might also have:

- Analyzed the role of silence in the tutoring interactions. As Johnstone (2002) noted, "The worlds that shape and are shaped in discourse involve absences as well as presences. Noticing silences, things that are not present, is more difficult than noticing things that are present, but it is equally important" (p. 58). When given time, students often came around to an answer that worked for both of us. In fact, a full $15 \%$ of appropriate student responses immediately followed substantive pauses in the interaction. A further analysis of the link between silence and other moves would, no doubt, have offered deeper insight.

- Explored more fully the role of power dynamics. This is a concern because, as Lefstein (2010) noted, "power relations are implicated in all human intercourse, and . . serve some interests while harming others" (p. 179). There was some evidence that I assumed more power than necessary, as when I interrupted a student as in the following example:

Bella: Like they, um, my opinion is that they like to, they like

Elizabeth: Who's they? The kids?

or rejected a response, in a way which may have served to dominate as well as to correct (Razfar, 2005):

Bella: A tide is like there's a little hole and there's water in it?

Elizabeth: Nope, let's see.

Three approaches appeared to foster a more equal and collaborative relationship. The first involved employing more tentative language. Rather than agreeing or disagreeing forcefully, I said, "In a way," after a not fully accurate response. Another approach involved the use of supportive language. This response took the form of acknowledging difficulty-"That IS a challenging word." Finishing my sentences with, for example, "Right?" or "OK?" was a third approach that prompted the students to self-assess their understanding or evaluate the text they were reading. But whether these moves affected the inherent power differential in any substantive way remains unknown.

- Coded specifically for tutor or student emotions. There were times when I expressed frustration and children showed evidence of lethargy or delight. Occasionally, students said or did something wholly unexpected such as the day Bella requested that we read more difficult books or usually-solemn Sam laughed so hard during a retelling that he had to take time to compose himself before moving forward.

\section{Implications}

Despite these limitations, the findings reported here have potential implications for theory, practice and research. To my knowledge, this is the first time the theoretical construct of instructional dialogicality has been employed to frame a tutoring study. What dialogue theory brings to tutoring research is a focus on the less tangible aspects of interaction in the tutoring context. Without doubt, the content of this intervention was important, but dialogue theory also considers (a) the structures of linguistic interaction; (b) the way in which speakers not only respond to what others say but also "take into account how they think 
other people are going to respond to them" (Wegerif, 2008); (c) the expression of tension, and (d) the drive for collaborative meaning-making. These are the aspects that surface in an analysis of tutoring discourse.

There are also implications for practice. All else being equal, it behooves a tutor to consider a question or strategy demonstration as the first line of response to student difficulty because these replies tended to lead to more productive dialogue than did providing information. Ensuing turns should demonstrate both variety and balance; any effort to avoid doing one thing over and over-questioning, information giving, or modeling -is worth the extra effort. It is also important to consider that, under some circumstances such as dealing with an unknown vocabulary word, simply providing the student with an answer and moving on is the best approach; lengthening dialogue sequences as an attempt to avoid "telling" may be counter-productive. Maintaining positive morale is a worthy goal, but, as evidenced by the excerpts included here, a balance of more and less successful instructional sequences appears adequate; alternating work on more and less challenging topics may prove effective. Because individual children respond differently to different moves, learning as much as possible about the child is beneficial. Bella, for example, was well-tuned to the physical environment around her, so it was no surprise that she responded especially well to visual cues. Regarding teacher education, providing pre-service and practicing teachers with tutoring opportunities and feedback—as occurred in the study by Timmons and Morgan (2010)—will support teachers in crafting productive interactions with students.

Finally, there are implications for future research. This study presents, of course, only half of the picture of tutor/student interaction. Analysis of data on response to student success would supplement that on student difficulty; tutors need to understand what moves occurring after an effective and/or interesting response from the child are most likely to further rich discussion. As noted in the limitations section, I did not investigate evidence of power dynamics. Such an investigation would contribute to the critical discourse analysis literature, as well as exploring this important aspect of the tutoring relationship. A replication study might include video-recordings of tutoring sessions so that body language and other non-linguistic forms of communication could be analyzed. Finally, future research might investigate the effects of teaching tutors to analyze audio- or video-recordings of their tutoring sessions, selecting one area of improvement they wish to work on, and noting the apparent influence of those changes on the relationships they build with the children they serve. As Wells (1996) argues, when teachers are aware of the dialogues in which they participate, they can change instruction for the better.

\section{References}

Alexander, R. (2004). Towards dialogic teaching: Rethinking classroom talk. Cambridge, UK: Dialogos.

Almazrouhi, K.M. (2007). Learning together through retrospective miscue analysis. Reading Improvement, 44 (3), 153-168.

Applegate, M.D., Quinn, K.B., \& Applegate, A.J. (2008). The critical reading inventory (2 ${ }^{\text {nd }}$ ed.). Upper Saddle River, NJ: Pearson.

Bakhtin, M.M. (1973). Problems of Dostoevsky's poetics. Trans. R.W. Rotsel. Ann Arbor, MI: Ardis.

Bakhtin, M.M. (1986). Speech genres and other late essays. Trans. V.W. McGee. Austin, TX: University of Texas Press.

Baumann, J.F., Jones, L.A., \& Seifert-Kessel, N. (1993). Monitoring reading comprehension by thinking aloud (Instructional Resource \# 1). Athens, GA: National Reading Research Center.

Boyd, M.P. (2015). Relations between teacher questioning and student talk in one elementary ELL classroom. Journal of Literacy Research, 47 (3), 370-404. 
Boyd, M.P., \& Markarian, W.C. (2015). Dialogic teaching and dialogic stance: Moving beyond interactional form. Research in the Teaching of English, 49 (3), 272-296.

Buly, M.R., \& Valencia, S.W. (2002). Below the bar: Profiles of students who fail state reading assessments. Educational Evaluation and Policy Analysis, 24 (3), 219-239.

Cameron, D. (2001). Working with spoken discourse. London, UK: Sage.

Chazan, D., \& Ball, D. (1999). Beyond being told not to tell. For the Learning of Mathematics, 19 (2), 2-10.

Clay, M.M. (1987). Learning to be learning disabled. New Zealand Journal of Educational Studies, 22 (2), 155-173.

Cohen, P.A., Kulik, J.A., \& Kulik, C-L.C. (1982). Educational outcomes of tutoring: A meta-analysis of findings. American Educational Research Journal, 19 (2), 237-248.

Constas, M.A. (1992). Qualitative analysis as a public event: The documentation of category development procedures. American Educational Research Journal, 29 (2), 353-366.

Cromley, J.G. (2005). What do reading tutors do? A naturalistic study of more and less experienced tutors in reading. Discourse Processes, 40 (2), 83-113.

Dawes, E.T. (2007). Constructing reading: Building conceptions of literacy in a volunteer read-aloud program. Language Arts, 85 (1), 10-19.

Edwards, T. (1992). Teacher talk and pupil confidence. In K. Norman, (Ed.), Thinking voices: The work of the National Oracy Project (pp. 235-241). London, UK: Hodder and Stoughton.

English, A.R. (2016). Dialogic teaching and moral learning: Self-critique, narrativity, community, and "blind spots." Journal of Philosophy of Education 50 (2), 160-176.

Fitts, S., \& Gross, L. (2012). Teacher candidates learning from English learners: Constructing concepts of language and culture in Tuesday's Tutors after-school program. Teacher Education Quarterly, 39 (4), 75-95.

Gee, J.P. (2011). How to do discourse analysis: A tool kit. New York, NY: Routledge.

Gibbs, G.R. (2007). Analyzing qualitative data. Los Angeles, CA: Sage.

Halliday, M.A.K. (1978). Language as social semiotic: The social interpretation of language and meaning. London, UK: Edward Arnold.

Hedin, L.R., \& Gaffney, J.S. (2013). Tutoring sixth graders who struggle with reading: Illustrations of Woods's contingent intervention. Reading Psychology, 34 (3), 207-256.

Herppich, S., Wittwer, J., Nuckles, M., \& Renkl, A. (2016). Expertise amiss: Interactivity fosters learning but expert tutors are less interactive than novice tutors. Instructional Science, 44, 205-219.

Howe, C., \& Abedin, M. (2013). Classroom dialogue: A systematic review across four decades of research. Cambridge Journal of Education, 43 (4), 325-356.

Jacob, E. (1987). Qualitative research traditions: A review. Review of Educational Research, 57 (1), 1-50.

Jaeger, E.L. (2015). Learning to construct meaning from text: A case study of the relationship between a tutor and an English learner in a Response to Intervention setting. Literacy Research and Instruction, 54 (4), 285-315.

Jaeger, E.L. (2017). Learning through responsive and collaborative mediation in a tutorial context. Australian Journal of Language and Literacy, 40 (3), 210-224. 
Johnstone, B. (2002). Discourse analysis. Malden, MA: Blackwell.

Juel, C. (1996). What makes tutoring effective? Reading Research Quarterly, 31 (3), 268-289.

Kohn, A. (1993). Choices for children: Why and how to let students decide. Phi Delta Kappan, 75 (1), 816 \& 18-20.

Leal, D., Johanson, G., Toth, A., \& Huang, C-C. (2004). Increasing at-risk students' literacy skills: Fostering success for children and their preservice reading endorsement tutors. Reading Improvement, 41 (2), 75-96.

Lee, Y-A. (2007). Third turn position in teacher talk: Contingency and the work of teaching. Journal of Pragmatics, 39, 180-206.

Lefstein, A. (2010). More helpful as problem than solution: Some implications of situating dialogue in classrooms. In K. Littleton \& C. Howe (Eds.), Educational dialogues: Understanding and promoting productive interaction (pp. 170-191). London, UK: Routledge.

Lewis, C. (1993). "Give people a chance": Acknowledging social differences in reading. Language Arts, 70 (6), 454-461.

Massey, D.D. (2007). "The Disney Channel said so" and other barriers to comprehension. The Reading Teacher, 60 (7), 656-666.

Matusov, E. (1996). Intersubjectivity without agreement. Mind, Culture, and Activity, 3 (1), 25-45.

Matusov, E. (2009). Journey into dialogic pedagogy. New York, NY: Nova Science Publishers.

Matusov, E. (2011). Authorial teaching and learning. In E.J. White \& M.A. Peters (Eds.), Bakhtinian pedagogy: Opportunities and challenges for research, policy, and practice in education (pp. 2146). New York, NY: Peter Lang.

Mehan, H. (1979). Learning lessons: Social organization in the classroom. Cambridge, MA: Harvard University Press.

Mercer, N. (1995). Guided construction of knowledge. Clevedon, UK: Multilingual Matters.

Mercer, N. (2004). Sociocultural discourse analysis: Analysing classroom talk as a social mode of thinking. Journal of Applied Linguistics, 1 (2), 137-168.

Mercer, N. (2014). The study of talk between teachers and students from the 1970s to the 2010s. Oxford Review of Education, 40 (4), 430-445.

MindTools (2017). SMART goals: How to make your goals achievable. Accessed from https://www.mindtools.com/papers/article/smart-goals.htm

Mokhtari, K., Hutchison, A.C., \& Edwards, P.A. (2010). Organizing instruction for struggling readers in tutorial settings. The Reading Teacher, 64 (4), 287-290.

Newcomer, L.J. (2010). An examination of instructional interactions between volunteer-tutors and students who show differential gains in reading comprehension (Doctoral dissertation). Available from ProQuest Dissertations and Theses database. (UMI No. 3402462)

Nystrand, M., Wu, L.L., Gamoran, A., Zeiser, S., \& Long, D.A. (2003). Questions in time: Investigating the structure and dynamics of unfolding classroom discourse. Discourse Processes, 35 (2), 135-198.

Pearson, P.D., \& Gallagher, G. (1983). The gradual release of responsibility model of instruction. Contemporary Educational Psychology, 8, 112-123. 
Pinnell, G.S., Lyons, C.A., Deford, D.E., Bryk, A.S., \& Selzer, M. (1994). Comparing instructional models for the literacy education of high-risk first graders. Reading Research Quarterly, 29 (1), 8-39.

Razfar, A. (2005). Language ideologies in practice: Repair and classroom discourse. Linguistics in Education, 16, 404-424.

Rodgers, E.M. (2004/2005). Interactions that scaffold reading performance. Journal of Literacy Research, 36 (4), 501-532.

Sableski, M-K. (2009). Scaffolding as an impetus for change when working with struggling readers. Journal of Reading Education, 34 (3), 30-37.

Sacks, H., Schegloff, E.A., \& Jefferson, G. (1974). A simplest systematics for the organization of turntaking for conversation. Language, 50, 696-735.

Samson, J.F., Hines, S.J., \& Li, K. (2015). Effective use of paraprofessionals as early intervention reading tutors in grades K-3. Mentoring and Tutoring: Partnerships in Learning, 23 (2), 164-177.

Sanacore, J. (1999). Encouraging children to make choices about their literacy learning. Intervention in School and Clinic, 35 (1), 38-42.

Shenderovich, Y., Thurston, A., \& Miller, S. (2016). Cross-age tutoring in kindergarten and elementary school settings: A systematic review and meta-analysis. International Journal of Educational Research, 76, 190-210.

Sinclair, J.M., \& Coulthard, R.M. (1975). Towards an analysis of discourse: The English used by teachers and pupils. Oxford, UK: Oxford University Press.

Timmons, B.J., \& Morgan, D.N. (2010). Preservice tutors and first-grade students: Instruction interactions and faculty feedback. Literacy Research and Instruction, 50 (1), 15-30.

Triplett, C.F. (2004). Looking for a struggle: Exploring the emotions of a middle school reader. Journal of Adolescent and Adult Literacy, 48 (3), 214-222.

Turner, J., \& Paris, S. (1995). How literacy tasks influence children's motivation for literacy. The Reading Teacher, 48 (8), 662-673.

Wegerif, R. (2008). Reason and dialogue in education. In B. van Oers, W. Wardekker, E. Elbers, \& R. van der Veer (Eds.), The transformation of learning: Advances in Cultural-Historical Activity Theory (pp. 273-286). Cambridge, UK: Cambridge University Press.

Wegerif, R. (2011). Towards a dialogic theory of how children learn to think. Thinking Skills and Creativity, 6, 179-190.

Wells, G. (1993). Reevaluating the IRF sequence: A proposal for the articulation of theories of activity and discourse for the analysis of teaching and learning in the classroom. Linguistics and Education, 5, $1-37$.

Wells, G. (1996). Using the tool-kit of discourse in the activity of learning and teaching. Mind, Culture, and Activity, 3 (2), 74-101.

Wells, G. (1999). Dialogic inquiry: Towards a sociocultural practice and theory of education. Cambridge, UK: Cambridge University Press.

Wittwer, J. Nuckles, M., Landmann, N., \& Renkl, A. (2010). Can tutors be supported in giving effective explanations? Journal of Educational Psychology, 102 (1), 74-89. 
Wood, D. (1992). Teaching talk. In K. Norman (Ed.), Thinking voices: The work of the National Oracy Project (pp. 203-214). London, UK: Hodder and Stoughton for the National Curriculum Council.

\section{(c) $)$ EY}

New articles in this journal are licensed under a Creative Commons Attribution 4.0 United States License.

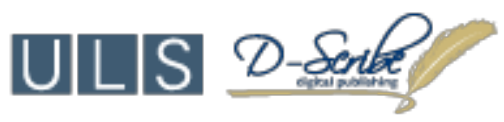

This journal is published by the University Library System, University of Pittsburgh as part of its D-Scribe Digital Publishing Program and is cosponsored by the University of Pittsburgh Press. 